\title{
Reattachment of Crown in Anterior Fractured Tooth-A Case Report
}

\author{
Jalison Jacob ${ }^{1}$, Abin Varghese ${ }^{2}$, Tina Varghese ${ }^{3}$, Philip Pradeep ${ }^{*}$, Bibin John 5 , Jissy George \\ ${ }^{1}$ Lecturer, Conservative Dentistry and Endodontics Department, Penang International Dental College, Malaysia. \\ ${ }^{2}$ Lecturer, Oral and Maxillofacial Surgery Department, Penang International Dental College, Malaysia. \\ ${ }^{3}$ Lecturer, Periodontology Department, Penang International Dental College, Malaysia \\ ${ }^{4}$ Lecturer, Conservative Dentistry and Endodontics Department, Penang International Dental College, Malaysia \\ ${ }^{5}$ Private Practitioner, Conservative Dentistry and Endodontics, India. \\ ${ }^{6}$ Private Practitioner, Oral Pathology, India.
}

*Corresponding Author: Philip Pradeep Lecturer, Conservative Dentistry \& Endodontics, Penang International Dental College, Penang, Malaysia.

Received Date: September 16, 2021; Accepted Date: September 27, 2021; Published Date: September 30, 2021

Citation: Jalison Jacob, Abin Varghese, Tina Varghese, Philip Pradeep, Bibin John, Jissy George, Reattachment of Crown in Anterior Fractured Tooth-A Case Report, J Dentistry and Oral Maxillofacial Surgery. 4(1) DOI: 10.31579/2643-6612/021

Copyright: () 2021 : Philip Pradeep, this is an open-access article distributed under the terms of the Creative Commons Attribution License, which permits unrestricted use, distribution, and reproduction in any medium, provided the original author and source are credited.

\begin{abstract}
In dentistry, traumatic injuries are most common in the anterior teeth due to its anatomic position. The various treatment modalities have been used depending on the extent of injury on teeth and supporting structures. Esthetic concerns are the most important challenges in modern dental practice. Adhesive dentistry is an excellent approach regarding esthetics, especially for fractured anterior teeth. This case report describes the successful treatment of reattachment procedure in a 17-year-old male patient presented with a fractured maxillary left central incisor.
\end{abstract}

Keywords: adhesive dentistry, fractured tooth, reattachment, trauma

\section{Introduction}

Several patients report to a dental clinic with pain caused due to trauma from an accidental fall, a traffic accident, contact sports or other outdoor activities. And amongst these reported patients, the most frequently seen traumatic injuries involve the fractures of the maxillary anterior teeth. Such traumatic injuries cause aesthetic, functional and phonetic problems. In children and adolescence, coronal fractures of the anterior teeth often occur due to their susceptible position in the oral cavity [1]. The various treatment modalities depend on the extent of the traumatic injury on the teeth and the supporting structures [2]. With the progressive advances of biomaterials used in adhesive dentistry, clinicians can re-attach a broken tooth fragment to the remaining tooth structure mechanically and chemically [3]. Nonetheless, this treatment can only be administered if the broken tooth fragment is available in a viable condition for reattachment to the remaining tooth structure [2]. Other treatment options include Resin Based composite or full- coverage crown but these procedures are not as conservative, esthetic or cost effective in comparison to the tooth reattachment procedure $[1,2,4]$. Therefore, this article describes a case report with coronal tooth fracture of the maxillary anterior tooth that was successfully treated using tooth fragment reattachment procedure.

\section{Case Report}

A 17-year-old male patient presented with a fractured maxillary left central incisor caused due to trauma from a fall. On clinical examination, the trauma was classified as an enamel/dentin fracture without pulpal exposure. The fractured tooth was found to be vital and exhibited no mobility. The broken tooth fragment was retained by the patient in a paper tissue and was examined if the fragment could be adapted favorably to the remaining tooth structure. The margins of the broken tooth structure did not exhibit any gross deformations. The fractured fragment was disinfected with $\mathrm{NaOH}$ and rinsed thoroughly with water.

Rubber dam was placed to isolate the fractured tooth. Tooth dentin and fractured part dentin was cleaned with a tungsten carbide bur. After beveling the enamel surface using diamond burs, etching of both the remaining tooth surface and the fragment was carried out for 15 seconds using $37 \%$ phosphoric acid then rinsed thoroughly with water. Then single Bond Universal Adhesive $\left(3 \mathrm{M}^{\mathrm{TM}}\right)$ was applied on the etched surfaces and light cured for 20 seconds using a light curing unit (Blue phase N MC, Ivoclar Vivadent). Universal Nano-Ceramic Restorative CeramX (DENTSPLY) was then applied to the opposing surfaces and the 
fragment was fitted to the remaining tooth. Polymerization was carried out from both buccal and lingual directions for 20 seconds. Finishing and polishing procedures were performed with Sof-Lex ${ }^{\mathrm{TM}}$ disks (3M ESPE). The repaired area was barely visible, and the esthetic result was excellent.

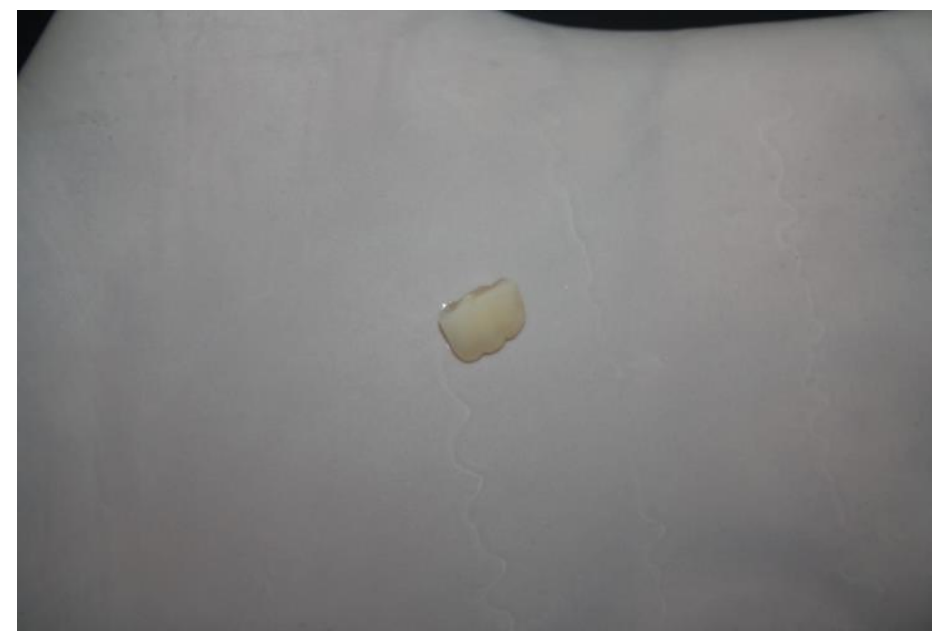

Figure 1

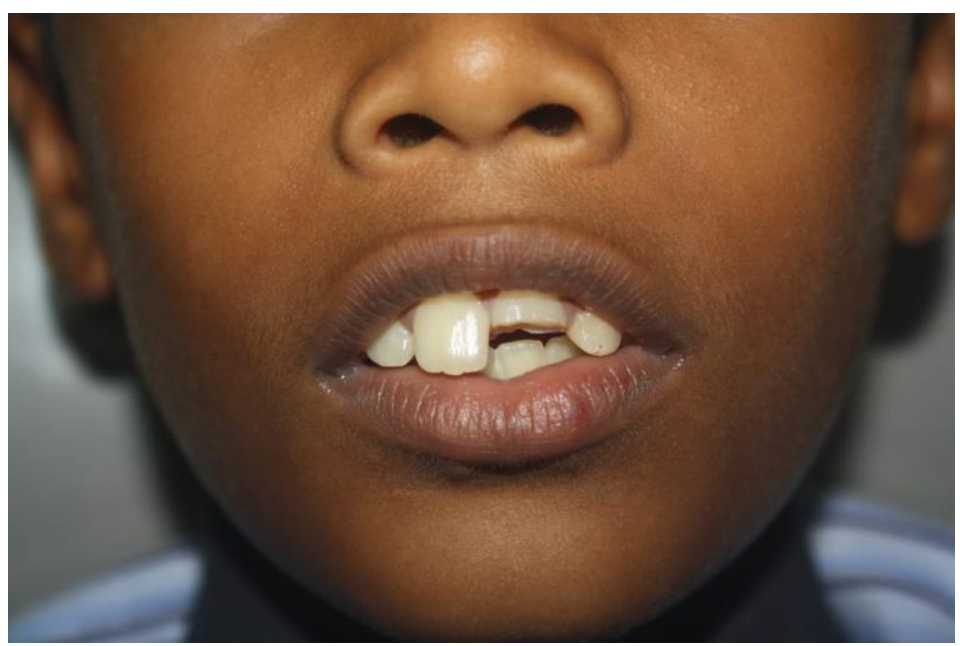

Figure 2

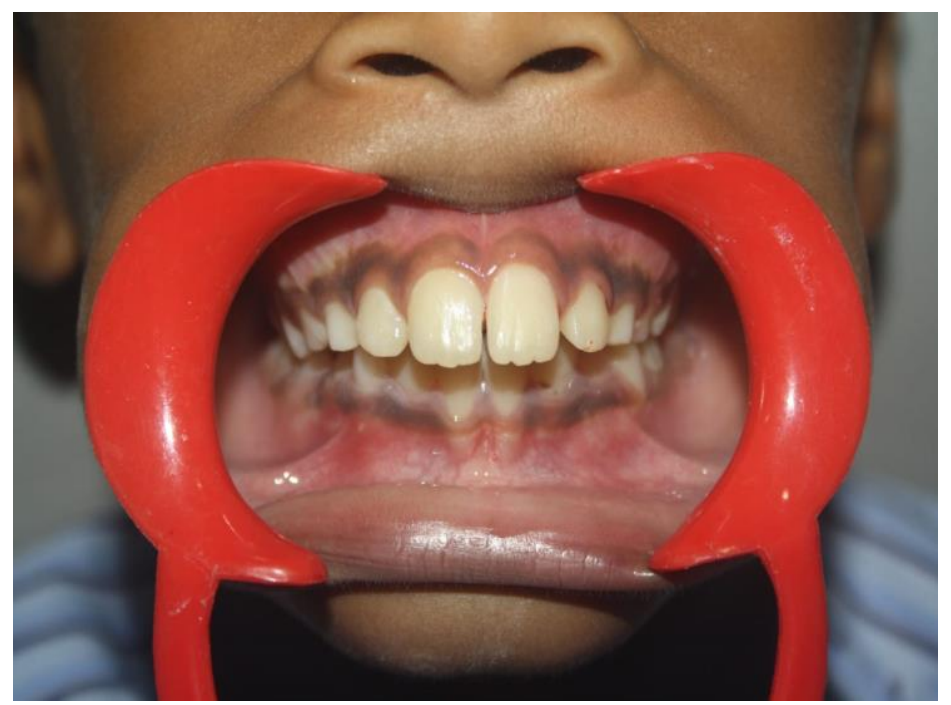

Figure 3 


\section{Discussion}

Loss of the coronal part of a permanent incisor in a young patient can cause esthetic and functional problems, which in turn can lead to severe emotional problems [4]. The treatment and prognosis for each case may differ according to the patient's age, amount of enamel available for bonding, wideness and wetness of the dentin tubules in young permanent teeth, possibility of bacterial contamination of the dentin and pulp, and availability of the tooth fragment for adhesion [5].

Numerous factors play an important role in determining how long the reattached tooth fragment remains functional. Among these factors, the media used to store the tooth fragment after fracture, type of material used for adhesion, use of materials to protect the dentin-pulp complex, flow of composite resins or cements, and technique used for the reattachment procedure are the most prominent [5,6,7]. However, recent studies have reported that different resin materials do not influence the fracture strength of the reattached tooth fragment $[6,2]$.

Some techniques of fragment reattachment include a bonding procedure without any type of wearing of the remaining tooth or tooth fragment surfaces. This technique is called simple reattachment [8].

The techniques described in these case reports are reasonably simple while restoring function and esthetics with a very conservative approach. However, the professional has to keep in mind that a dry and clean working field and the proper use of bonding protocol and materials is the key for achieving success in adhesive dentistry.

The following re-attachment strategies have been advocated for reattaching a detached tooth fragment to the remaining tooth:

- Placement of a circumferential bevel before re-attaching the fragment

- Placement of an external chamfer at the fracture line after bonding

- Use of a V-shaped enamel notch

- Placement of an internal groove

- Leaving a superficial over contour of restorative material over the fracture line $[4,9]$

Limited data is available on the strength of reattached fractured fragments [8]. In this case, an internal groove was prepared in the tooth fragment surface. This groove had two objectives: (1) creating physical space for the material protecting the dentin-pulp complex and composite resin cement and (2) increasing the fracture strength of the reattached tooth fragment. Consequently, an accurate adaptation of the tooth fragment was achieved, and esthetic, functional, and biological parameters could be successfully maintained for 10years [8, 10, 11].

Although some authors have reported the absence of clinical discolorations and pathological changes in the anterior teeth with an original fragment reattached, the follow-up duration of these cases were
24 and 12 months, respectively [10,11,12]. This period can be considered short for evaluating the clinical success of some treatments, such as tooth fragment reattachment, in young people. Thus, an immediate tooth fragment reattachment should not be considered as a temporary alternative treatment for fractured anterior teeth just because it offers an excellent restorative option for clinicians and patients $[10,12]$.

\section{Conclusion}

Reattaching a tooth fragment with self-etching adhesives may be successfully used to restore fractured teeth with adequate strength, but long-term follow up is necessary in order to predict the durability of the tooth-adhesive-fragment complex and the vitality of the tooth.

\section{References}

1. Mazumdar P., et al. (2015). Management of horizontal and oblique crown fracture of anterior teeth by reattachment using fiber post: a case report. International Journal of Advances in Case Reports. 2(1):5-8.

2. Mendes L et al. (2017). Ten-Year Follow-Up of a Fragment Reattachment to an Anterior Tooth: A Conservative Approach. Hindawi. Case Reports in Dentistry.

3. EmreOzel (2008). Re-attachment of Anterior Tooth Fragment using a Self-etching Adhesive: A Case Report. The Journal of Contemporary Dental Practice. 9(1).

4. Macedo et al (2008). Reattachment of Anterior Teeth Fragments: A Conservative Approach Journal compilation. 20(1).

5. Pamir T et al. (2012). "Shear bond strength of restorations applied to un-complicated crown fractures: An in vitro study." Dental Traumatology. 28(2). pp. 153-157.

6. Chazine $\mathrm{M}$ et al. (2011). "Evaluation of the fracture resistance of reattached incisal fragments using different materials and techniques.” Dental Traumatology. 27(1) pp. 15-18.

7. Prabhakar A.R et al. (2016). "Effect of storage media on fracture resistance of reattached tooth fragments using G-aenial Universal Flo." Journal of Conservative Dentistry. 19(3). pp. 250-253.

8. Abdulkhayum A et al. (2014). "In-vitro evaluation of fracture strength recovery of reattached anterior fractured tooth fragment using different re-attachment techniques." Journal of Clinical and Diagnostic Research. 8(3). pp. 208-211.

9. Yilmaz Y et al. (2008). "Evaluation of success in the reattachment of coronal fractures." Dental Traumatology. 24(2) pp. 151-158.

10. Pavone $\mathrm{M}$ et al. (2016). "Autogenous tooth fragment adhesive reattachment for a complicated crown root fracture: two interdisciplinary case reports," Case Reports in Dentistry.

11. Gutierrez O. (2011). "Reattachment of anterior teeth fragments using a modified Samisen's technique after dental trauma: report of a case". Dental Traumatology. 27(1). pp. 81-85.

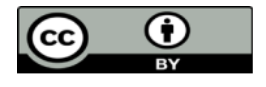

This work is licensed under Creative Commons Attribution 4.0 License

To Submit Your Article Click Here: Submit Manuscript

DOI: $10.31579 / 2643-6612 / 021$
Ready to submit your research? Choose Auctores and benefit from:

$>$ fast, convenient online submission

$>$ rigorous peer review by experienced research in your field

$>$ rapid publication on acceptance

$>$ authors retain copyrights

$>$ unique DOI for all articles

$>$ immediate, unrestricted online access

At Auctores, research is always in progress.

Learn more https://auctoresonline.org/journals/dentistry-and-oralmaxillofacial-surgery 